\title{
UN HOMBRE ENAMORADO DEL PASADO: LAS CRÓNICAS DE ANTONIO MARICHALAR EN LA REVISTA THE CRITERION
}

\section{MODERNIDAD Y CRÍTICA LITERARIA}

Las recientes lecturas e interpretaciones en torno a la cultura española de las tres primeras décadas de nuestro siglo coinciden en plantearla como un proceso sin centros. A partir de dicha hipótesis se prefiere hablar de "modernidad literaria", y a la par que se hace más permeable la cronología se intentan establecer los necesarios puentes entre las diversas "modernidades" europeas. Transeúnte habitual de esos puentes fue Antonio Marichalar, Marqués de Montesa, que por su prosa crítica y su condición de intermediario entre diversas culturas europeas, nos parece una figura imprescindible de la llamada "modernidad literaria". Período para el que nos sigue pareciendo sensata la síntesis de Ilustración y Romanticismo, inteligencia crítica y sensibilidad inquieta, con la que Cyril Connolly define, en un sentido más amplio, el espíritu moderno: "a combination of certain intellectual qualities inherited from the Enlightenment: lucidity, irony, scepticism, intellectual curiosity, combined with the passionate intensity and enhanced sensibility of the Romantics, their rebellion and sense of technical experiment, their awareness of living in a tragic age"1.

En la distancia, Montesa se nos presenta como un cabal representante de la generación del 14, colaborador de $\mathrm{El} \mathrm{Sol,} \mathrm{la}$ Revista de Occidente, y más tarde, Cruz y Raya. De cerca se revela como un escritor exigente con su prosa, trabajador de la tra-

1100 key books of the modern movement from England, France $\mathcal{E}$ America, 1800-1950, Allison \& Busby, London, 1986, p. 11. Lamentablemente este brillante crítico retrocede lo avanzado por Eliot en cuanto a cosmopolitismo, ya que sólo incluye libros ingleses, franceses y norteamericanos en su "canon" de la modernidad. 
dición y autor de algunas de las páginas más interesantes de la crítica literaria y artística de su tiempo, páginas escritas en una prosa que proviene de ese sueño de perfección y profundidad que caracterizó a los hombres del 14 y del que serán depositarios los ensayistas del 27.

En el retrato que de él hiciera Juan Ramón se reparte una misma cosecha de elegancia y solidez en dos tiempos: la infancia ("una pequeña conciencia esbelta") y la madurez ("un hombre cuajado"). La primera, pasada en cierta esquina ilustrada del Madrid del cambio de siglo, Puerta de Alcalá y Retiro, marca arquitectónicamente al adulto "especialista de la prosa" en cuyo estilo se funden "materiales de orden, de armonía, de encaje" 2 . Es la imagen de un clásico moderno, moderno en el sentido anglosajón del adjetivo: lector de todas las tradiciones desde el momento en que "all ages are contemporaneous", según la afirmación de Pound en su ensayo The spirit of romance (1910).

La contemporaneidad de todas las épocas es un suceso histórico que acontece en algunos lugares de Europa hacia los años veinte y que conllevará una determinada concepción de la crítica artística y literaria. La evolución de la prosa crítica de Marichalar es, precisamente, un ejemplo de la evolución de ese tipo de modernidad estética. Los pensamientos literarios del modernism anglosajón y de la vanguardia española comparten un conjunto de soluciones a los problemas estéticos y éticos de los años veinte. En concreto, quienes comparten esas soluciones son el modernism que se desprende de la revista The Criterion y los ensayos de Eliot y Pound, y la vanguardia española que se debate en la Revista de Occidente y los ensayos de Ortega y Marichalar, entre otros. Porque, en realidad, las reacciones intelectuales y artísticas frente a la deshumanización y la cultura de masas son mucho más variadas.

El problema para Marichalar, como para Eliot y Ortega, es la relación crítica con la novedad y con la tradición, esto es, la elaboración de un método que permita relacionarse de igual manera con lo reciente y con lo pasado en materia de arte. Una crítica que aspira a tal nivelación de tiempos implica, por tanto, un sentido de la tradición, pero no necesariamente un sen-

${ }^{2}$ El retrato de Juan Ramón se encuentra en la edición de Ricardo Gullón de Españoles de tres mundos, de Juan Ramón Jiménez, Afrodisio Aguado, Madrid, 1960, pp. 208-209. 
tido histórico. En efecto, la modernista contemporaneidad de todas las épocas equivale en gran medida a una eliminación de la historia ${ }^{3}$.

Ese es el sistema crítico que vertebra las colaboraciones de Marichalar en The Criterion entre 1923 y 1938: la actualidad y la tradición, esta última en forma de conmemoraciones o relecturas, descansan en el mismo horizonte fenomenológico, incluso, utilizando una terminología más reciente, en el mismo "horizonte de expectativas". No es de extrañar que la evolución de sus preocupaciones intelectuales evidencie un paulatino desvío hacia la reescritura de la tradición en forma de biografías. Así, antes de la guerra, cuando todavía la modernidad se vive desde la estética, su prosa gira en torno a la crítica de arte y de la literatura (Girola, críticas de arte, 1926; Mentira desnuda, críticas literarias publicadas en su mayoría en la Revista de Occidente, 1933); después del 39 sus preocupaciones intelectuales se orientarán hacia el ensayo histórico y la biografía (Riesgo y ventura del duque de Osuna, 1930, como precedentes; luego vendrán Tres figuras del siglo xvi, 1945, Las cadenas del duque de Alba, 1947, y Julián Romero, 1952).

A partir de su primera etapa, es decir, aquella que gravita alrededor de la Revista de Occidente, Soria Olmedo ha intentado definir el sistema crítico de Marichalar como individualista, antirromántico y antisurrealista. Para sostener la definición de dicho sistema Soria echa mano de un artículo de Marichalar publicado en la cubana Revista de Avance en el que el de Montesa equipara a crítico y artista: "El crítico es artista, a su vez, por cuanto a él corresponde recrear al artista, deduciéndolo del acervo general de sus obras" 4 . En el mismo sentido, desmarcándose de la crítica informativa y pedagógica, llegará a afirmar en otro momento que "Para poder ser imparcial es necesario

${ }^{3}$ Precisamente las vinculaciones entre modernidad y literatura, y modernidad e historia, son las que cuestiona Paul de Man: "no hay certeza alguna de que la literatura y la modernidad sean conceptos de alguna manera compatibles", afirma por un lado mientras que por otro echa mano de un famoso texto de Nietzsche ("Vom Nutzen und Nachteil der Histoire für das leben") para poner en duda el sentido histórico de la modernidad, "Historia literaria y modernidad literaria", Visión y ceguera. Ensayos sobre retórica de la crítica contemporánea, Universidad de Puerto Rico, Río Piedras, 1991.

${ }^{4}$ El artículo de Marichalar, "El arte de la crítica", en Revista de Avance, 30 de octubre de 1927, núm. 14, 48-50; cf. A. Soria Olmedo, Vanguardismo y crítica literaria en España, Istmo, Madrid, 1989, pp. 236-237. 
ser apasionado" . También se desmarcará de los objetivos no creativos de la crítica otro de los grandes del pensamiento literario de este siglo, el romanista E. R. Curtius quien en un ensayo sobre Eliot se apoya precisamente en el Marqués de Montesa para definir su concepción aventurera de la crítica, personificada en el crítico injertado en poeta, en la inteligencia que hace fiable la intuición ${ }^{6}$.

El modernismo eliotiano implica en poesía un proceso de impersonalización de la voz individual dispersándola entre las voces de la tradición, proceso que sintoniza en gran medida con el proyecto del moderno clasicismo español que ejemplificamos en Marichalar: el pasado histórico, la tradición, como lugar de búsqueda del sentido personal. Proceso que implica una paradoja: a mayor impersonalización mayor individualización de la actividad creativa. Sólo cuando el pasado se hace irrescatable, o el presente desaprende la posibilidad de actualizar, es cuando el proyecto del moderno clasicismo hace agua y enfila hacia posturas políticas reaccionarias. Es entonces cuando la prosa crítica de Marichalar se recluye en el ensayo histórico. Ese fracaso lo previó Ortega en sus Meditaciones del Quijote cuando advierte la imposibilidad del reaccionario para tratar el pasado como "un modo de la vida"7, para superar "the pastness of the past", en palabras de Eliot.

El recurso a la tradición entre los modernos es una manera de solventar lo que se vive como una posible catástrofe cultural: la modernización social y sus manifestaciones en el mundo del arte y la cultura, esto es, la deshumanización y la masificación. De hecho el concepto "modernismo", en el sentido supranacional del término, designa un heteróclito conjunto de respuestas artísticas y filosóficas protagonizadas por un grupo humano que tiene asumidos unos valores en conflicto con el proceso de modernización ${ }^{8}$. Las respuestas a ese cambio son extremas y consecutivas: ruptura con la tradición y actualización de ciertas tradiciones.

${ }^{5}$ Mentira desnuda. (Hitos), Espasa Calpe, Madrid, 1933, p. 97.

${ }^{6}$ Cf. "T. S. Eliot", Ensayos críticos acerca de literatura europea, Visor, Madrid, 1989, pp. 276-277.

${ }^{7}$ En Obras completas, Alianza Editorial-Revista de Occidente, Madrid, t. 1, p. 325 .

8 Así lo entiende R. SHEPPARD, "The problematics of European modernism”, en Theorizing modernism. Essays in critical theory, ed. S. Giles, Routledge, London-New York, 1993, pp. 1-51. 
Nos proponemos aquí releer y situar en su contexto las crónicas de la cultura española publicadas en una de las revistas literarias más relevantes de la Europa de entreguerras, período en el que Antonio Marichalar desempeña una función de agente doble entre España y Europa: mientras comenta la cultura española en The Criterion, escribe sobre las figuras más destacadas del modernismo europeo (Joyce, Valéry, Rilke) en la Revista de Occidente.

La elección de Marichalar como corresponsal de The Criterion en España corresponde a los criterios conservadores de Eliot y su tendencia a sintonizar con el lado tradicional y católico de la modernidad española ${ }^{9}$. Tendencia que encontramos, por ejemplo, en el elogio que se hace del prólogo de Marichalar a la traducción de $A$ portrait of the artist as a young man como el mejor posible dedicado a un país católico (The Criterion, V, núm. 1) ${ }^{10}$. Y es que para la dirección de The Criterion todo lo artísticamente relevante que procede de España tiene un sello católico. Léase, por ejemplo, la reseña de E. Muir a la traducción inglesa del Llanto por Ignacio Sánchez Mejías (XVII, núm. 66): "Their reason for doing so (executed by Franco's followers) cannot be discovered from this book, for Lorca seems to have been a traditional poet and a catholic... Perhaps Franco approved of the Moorish note in practical politics, but not in literature" (p. 153).

En el prefacio a la edición facsimilar de la revista, realizada en 1967, T. S. Eliot recuerda sus intenciones al poner en marcha The Criterion: presentar a los lectores ingleses por medio de ensayos o relatos cortos, el trabajo de los autores extranjeros más importantes, y ofrecer más largas y razonadas reseñas que las que aparecían en otras revistas. En cuanto a la orientación estética, está claro que por medio de la revista, Eliot quiere profundizar en la autonomía del arte literario, de modo que las notas de antirromanticismo y antisurrealismo, que veíamos en Marichalar, son las que impone Eliot desde la nota escrita al

${ }^{9}$ Cf. K. M. Sibbald \& H. T. Young, Eliot and the Hispanic modernists (19241993), Society of Spanish and Spanish-American Studies, Boulder, CO, 1994, p. 2.

10 Traducción de "Alfonso Donado" [Dámaso Alonso], Biblioteca Nueva, Madrid, 1926. En adelante citaré a The Criterion por tomo, número y página. 
cerrarse el primer volumen de la revista "The function of a literary review" (I, núm. 4): "It is the function of a literary review to mantain the autonomy and disinterestedness of literature... without tolerating any confusion of the purposes of pure literature with the purposes of politics or ethics" (p. 421).

Esta nota de Eliot junto a otra de Richard Aldington en el mismo número ("Literature and the Honnête homme", p. 422) conforman el credo estético y moral de los primeros momentos de la revista. La de Aldington plantea la oposición entre masas y aristocracia en cuanto a autoridad para juzgar las obras del espíritu. Enfoque tan familiar para nosotros que F. S. Flint no puede menos que resaltarlo en su reseña del primer número de la Revista de Occidente (II, núm. 5, p. 109): "True, great literature has been produced and still may be produced without any such society, but, lacking some such tradition of culture, literature is always liable to succumb to the provincial spirit, to fall into long arid periods of mediocrity and incompetence".

En otro momento Eliot menciona lo que para él es más una tendencia que un programa de la revista: el clasicismo como balanza en la que discriminar lo que cuenta y lo que no cuenta, lo prescindible de lo imprescindible, de modo que la revista adquiera un valor crítico y documental para la posteridad: "the bound volumes of the decade should represent the development of the keenest sensibility and the clearest thought of ten years" ("The idea of a literary review", IV, núm. 1, p. 2). Junto al moderno clasicismo la revista también ha de desarrollar, en opinión de Eliot, un moderno humanismo por medio del cual los avances científicos se hagan inteligibles y adquieran una dimensión humana. En consecuencia, a la creación y crítica literaria se añade cualquier material que sea operativo para las "ideas generales" de dicho humanismo: "the results of contemporary work in history, archaeology, anthropology, even of the more technical sciences when those results are of such a nature to be valuable to the man of general culture and when they can be made intelligible to him" (ibid., p. 4).

La tendencia clasicista y humanista que defiende Eliot conduce a una determinada manera de entender la política y la cultura que entonces empieza a llamarse "europeísmo". Para articular la comunión entre lo clásico y lo europeo Eliot no contempla mejor vehículo que el entramado internacional de las revistas literarias: la Revista de Occidente desde España, la Nouvelle Revue Française desde Francia, 900y Nuova Antologia desde Ita- 
lia, Neue Rundschau y Europäische Revue desde Alemania, Journal de Genève y Neu Schweizer Rundschau desde Suiza, y más allá de Europa The Dial desde Nueva York y Sur desde Buenos Aires. La red de opiniones y juicios que de sus ejemplares se desprende es lo que puede dar virtualidad a la unidad cultural europea: "the existence of such a network of independent reviews, at least one in every capital in Europe, is necessary, for the transmission of ideas"11.

De entre todas las publicaciones mencionadas la Revista de Occidente es la considerada más afín al proyecto europeísta y clasicista de The Criterion, y ello a pesar de que España como país de cultura no poseía mucho predicamento entre los lectores ingleses ${ }^{12}$. Pero a pesar de partir con desventaja, la revista de Ortega consigue situar la cultura española en el centro de la discusión europea, así se deduce al menos de los comentarios que le dedica J. B. Trend en junio de 1928 (VII, núm. 4) alabando su amplia idea de lo europeo, semejante a la que desde Alemania propone Curtius:

Under the able direction of Sr. Ortega y Gasset, this review continues to justify its reputation as one of the most broadminded in Europe. It is by no means confined to that "Near West" defended by M. Massis, nor yet to that mundo latino exalted by certain Spanish journalists, which includes the South American dependencies of Paris but excludes so "strongly Germanized" a country as Italy. Its general tendency seems to be that indicated by Ernst Robert Curtius, in his plea for the Restoration of Reason (p. 459) ${ }^{13}$.

En la misma reseña, y en el mismo contexto en el que se aboga por la construcción de la unidad europea, el comentarista habla de la modernidad, formal y espiritual, de los autos de Calderón. Lo que no deja de ser un síntoma más de las operaciones estéticas conducentes a construir un nuevo canon europeo.

La amplitud de miras y la inteligente capacidad para superar las barreras nacionales de la Revista de Occidente también es

11 T. S. ELIot, Notes towards the definition of Europe, Faber \& Faber, London, 1969, p. 116.

12 Véase la reseña de J. B. Trend al libro de Américo Castro, El pensamiento de Cervantes: "Contemporary English critics are not very ready to admit that Spain, as a country of the mind, has any real existence" (V, núm. 1, p. 146).

13 Para la articulación de esta vuelta al orden en la Revista de Occidente por medio de Curtius, véase A. Soria Olmedo, op. cit., pp. 158-159. 
objeto de admiración por parte de otro de los habituales en el comentario de la cultura española, F. S. Flint (II, núm. 5): “This is the most alertly intelligent of all foreign reviews that reach me: intelligent in the sense that its eyes are open on the world, the whole world; they are not directed solely to the doings of a particular clique or coterie or even nation".

La puesta al día del cosmopolitismo, con indumentarias de la autonomía del arte lleva unida una dilucidación sobre la tradición europea y el rechazo de la opinión de las minorías. Respecto a estas últimas, Eliot llega a afirmar que "instead of a few potential Sarajevos, we seem to have dozens" (VI, núm. 2, p. 97). En resumen, tanto Eliot como Ortega hacen suyo y trasladan a sus respectivas revistas el proyecto de Curtius para tratar de armonizar la idea alemana de cultura y la idea francesa de civilización, con los beneficios pero también con los peligros que esta unión pudiera comportar.

A la hora de acercarnos a los dieciocho volúmenes (19221939) de la revista de Eliot hay que tener presentes sus dos bien diferenciadas épocas. La primera comprendería los números publicados entre fines de 1922 y junio de 1928, fase que coincide con el arranque de la Revista de Occidente (1923) y se caracteriza por el europeísmo político y artístico al que acabamos de hacer referencia. La segunda época va de 1928 hasta su cierre en 1939, período en el que se aprecia un notable cambio: las crónicas del decenio 1920 están interesadas por las nuevas formas de expresión artística mientras que en las de 1930 las tendencias modernistas empiezan a ser tratadas con hostilidad. También es visible un cambio de configuración en el europeísmo pues a finales de la década de los treinta Eliot llega al convencimiento de que la civilización peligraba menos en manos del fascismo que del comunismo ${ }^{14}$. En un esclarecedor comentario sobre la España de Franco, el autor de The waste land afirma lo siguiente: "one might be persuaded to belive that the rebels... had finally and reluctantly taken to arms as the only way left in which to save Christianity and civilisation" (XVI, núm. 63, p. 289).

${ }^{14}$ Resulta inevitable recordar aquí el estudio sobre la cara fascista del "modernismo" de F. Jameson, Fables of aggression: Wyndham Lewis, the modernist as fascist, University of California Press, Berkeley, 1979. 
La relación de The Criterion con los literatos españoles no empieza con Marichalar sino con Ramón Gómez de la Serna. Las cuatro piezas de Ramón que bajo el título "From The New Museum" traduce F. S. Flint en el segundo número de la revista tienen una pequeña historia que resume los avatares de la puesta en marcha de The Criterion. Eliot pretendía contar con los más destacados representantes de las literaturas continentales, así que el primer número se hace con textos de Hermann Hesse, Valery Larbaud y Gómez de la Serna. En carta a E. R. Curtius (1 de julio de 1922) el propio Eliot describe el material que tiene preparado para el número inaugural: "You ask about the programme of the review. The first number will contain contributions by myself, Mr. George Saintsbury, Mr. T. Sturge-Moore, Miss May Sinclair and Mr. Richard Aldington in this country, and two or three foreign contributions - Gómez de la Serna, Valery Larbaud, and Hermann Hesse" 15.

El artículo de Gómez de la Serna queda fuera del plan del primer número y así se lo cuenta a $\mathrm{F}$. S. Flint, quien no debía tener una buena opinión sobre el autor español, en carta del 15 de agosto de 1922: "I am indeed sorry that you have been so harrassed. While I am not sure, without longer study, that I quite share your opinion of the author, I am at least doubtful whether it is quite what we want for the first number... I have another Spanish contribution, typed, and perfectly simple and straightforward chronique of Spanish letters, which I propose to substitute for the Gómez" (ibid., p. 559).

Las piezas de Ramón, traducidas en clave imaginista por Flint, se publicaron finalmente en el segundo número de la revista. Son un ejemplo de la nueva manera de mirar las cosas que caracteriza el sistema de Ramón: la vida secreta de las cosas, la animación de lo inanimado, las perspectivas que alteran la realidad, y sobre todo, la condición "vouyeurística" del artista moderno.

El artículo que iba a sustituirlo en el primer número es la crónica de Antonio Marichalar, "Contemporary Spanish literature", traducida por Mde. S. A. Middleton, y que también se retrasa hasta su publicación en el tercer número de abril de

15 V. Eцiot (ed.), The letters of T. S. Eliot, Faber \& Faber, London, 1988, t. 1 , p. 550 . 
1923. Marichalar abre sus crónicas inglesas con un repaso al estado de la literatura española a la altura de agosto de 1922, fecha en la que firma el texto. Los conjuntos y las ordenaciones vienen dadas por el esquema generacional. El silencio de Picón o de Palacio Valdés, representantes de la penúltima generación del siglo XIX, justifica su punto de partida en los autores del 98, cuyas obras son, en sus palabras, "a vehicle for the evolution of modern ideas" (p. 278). Marichalar presenta a los noventayochistas como reformadores de la tradición y del gusto literario, al tiempo que se preocupan por sintonizar con la cultura europea del momento. En ellos hay que situar, por tanto, el inicio de la "modernidad literaria" en España:

Very soon the writers of 1898 arrested public attention... reaffirming the true value of national feeling, revising classical writers, purifying and giving a new orientation to public taste, intensifying the cult of artists such as Góngora and Greco, obscure through lack of inappreciation; pointing out the intrinsic value of basic tradition and fostering it - while bringing them into touch with modern European thinkers- importing principally the works of Nietzsche, Ibsen, Tolstoy, Brandès, and A. France (pp. 277-278).

A la cabeza de ese movimiento renovador coloca a Unamuno, cuyo carácter egocéntrico, inconsecuente y paradójico es comparable al de Whitman. Especialmente interesante es el elogio que hace Marichalar de la paradoja unamuniana que plantea su dedicación a la política, actitud contradictoria que introduce elementos de avance y renovación en la sociedad española: "thanks to those who are capable of producing great paradoxes, many countries - England very especially - have visualised in the history of their ideas the rapid advancement of these" (p. 279). La más reciente obra de Unamuno, El Cristo de Velázquez (1920), es el pivote sobre el que Marichalar hace girar su interpretación del misticismo revolucionario y, lo que es más interesante para el lector inglés, del catolicismo heterodoxo que tantas semejanzas tiene con el que profesara el propio Eliot.

De Azorín, el que a su juicio más valores modernos posee, resalta su capacidad para reformar el lenguaje literario y, a partir de él, inventar un estilo "in appearance vulgar and drab, but very original, the kernel of which is true, delicate enchanting" (p. 280). La contrapartida del refinado Azorín es Baroja. Para Marichalar, el autor de Las ciudades no es una pista por seguir; sin embargo, destaca la condición de adelantado al entablar el 
diálogo entre procedimientos artísticos y principios sociales. Baroja es "the man who looks down upon the ground" (p. 281), cuyas novelas están construidas con un estilo neutro y una prosa de diagnosticador.

En Valle-Inclán sí señala el crítico a un "master of prose", a la par que resalta su preocupación por la forma y analiza su obra de acuerdo con una evolución estética que va de lo sensual a lo burlesco, de las "sonatas" al "esperpento". Del resto de noventayochistas menciona a Bueno, Cijes, Maeztu y Benavente, al que al final de la crónica dedicará un sorprendido post scriptum con motivo de la concesión del Premio Nobel. Es preciso interpretar la obra de los autores posteriores desde un punto de vista nacional, a partir de la brecha estética iniciada en el 98. Llegan entonces los que Marichalar denomina "young masters" del movimiento moderno en las letras españolas. La obra poética de Juan Ramón Jiménez es para Marichalar el mejor ejemplo de la dificultad moderna y de la depuración intelectual, "claridad dificultosa", a la que aspira el moderno clasicismo: "romantic and at the same time classical, uniting the intimate, emotional, personal, and popular qualities of his meridional temperament, creator of Becquer [sic.], to a masterly touch of exquisite refinement that strongly reminds one of Paul Valéry" (p. 284).

Marichalar pone de relieve en las obras de Jiménez y Machado el doble movimiento que caracteriza a la modernidad cuando entra en diálogo con la tradición de la poesía española y con las nuevas tendencias de la cultura europea: "After the manner of the first symbolists who revived popular poetry with their lyrical sentiment, essentially evocative and sympathetic - Juan Ramón Jiménez and Machado have oriented the modern lyric back towards its origin- legitimately spanish, mediaeval, and popular poetry, which has usually shown traces of foreign influences of a later date" (p. 285).

Sorprende que no muchas líneas más estén dedicadas a Antonio Machado. Es cierto que el moderno clasicismo al que se adscribe el de Montesa sigue la estela de Juan Ramón, pero Machado no dejaba de ser un claro referente para la joven generación.

Una vez establecido el magisterio de Juan Ramón, aparte de los dos Machado, Gabriel Alomar, Enrique de Mesa y Manuel Abril, distingue la figura de Enrique Díez Canedo, "modern humanist", en tanto que crítico, traductor e intermediario. La nó- 
mina de "jóvenes maestros" se completa con Pidal, Altamira, Cossío, Ortega, al que presenta naturalmente con la mayor amiración, Pérez de Ayala, d'Ors, Miró, Moreno Villa y los humoristas Camba, Fernández Flórez, Bello y Gómez de la Serna.

Pero Ramón Gómez de la Serna es más que un humorista también para el corresponsal español de The Criterion, es uno de los autores que más ha contribuido a la aceptación en España del arte moderno. Para Marichalar, Ramón tiene un afán de modernidad que, sin control, podría resultar peligroso: "so intensely modern did he show himself at a given moment that he might have endangered his continued advancement, but without losing his place he continues to keep abreast with the tide" (p. 288). Si bien destaca, en tanto que hallazgo, el funcionamiento de la analogía ramoniana que pone al descubierto inesperados aspectos psicológicos de la realidad, de las que el lector inglés tiene algunas pruebas en el anterior número de la revista, reprocha a Ramón su falta de capacidad para la síntesis y lo irreflexivo de su estilo, lo que no es óbice para que siga considerándolo un gran "modernizador" de la lengua literaria española.

A partir de ese momento de la crónica, cercana su conclusión, Marichalar se cura en salud y da su primer paso atrás. La prometida llegada a las fronteras de la actualidad no satisface las expectativas de los curiosos lectores. Sobre los que vienen después de Ramón no tiene una opinión cuajada pues, según él, no se puede opinar sobre lo que todavía no ha sucedido. Para ilustrar su postura recuerda al mono del Quijote: "I content myself, like the monkey of our Quixote, with knowing a little of the past and of the present, because what is yet to happen, says Cervantes, not even the devil himself really knows" (p. 292). No obstante, en la siguiente crónica sabrá algo más que el mono del Quijote. Quizá presionado por la orientación de la revista que, si seguimos a Eliot, quería estar al día y llevar a sus páginas la mejor literatura de cada país.

Hasta 1926 (IV, núm. 2) no vuelve a aparecer una crónica de Marichalar en The Criterion. Es posible que dejara pasar el tiempo suficiente para poder mandar a Londres su diagnóstico sobre la joven literatura española, su panorama de la, todavía en ciernes, "Generación del 27".

El de Montesa inicia la crónica de los del 27 afirmando el signo lírico de la nueva literatura española: "in Spain, the present age is undoubtedly passing through the sign of the lyric", afirmación que repetirá después Salinas en su ensayo "El signo 
de la literatura española del siglo xx" (1940). Además, Marichalar explica esa condición lírica por la minoría selecta, en el sentido orteguiano, que protagoniza en ese momento el movimiento literario español. Desde ese punto de vista comentará no lo que habitualmente se comenta, sino lo que debería comentarse y, en todo caso, lo que interesa en los "selectos" círculos que frecuentaría en España un lector de The New Criterion, que es como se llama la revista a partir de enero de 1926. Arranca comentando la concesión del Premio Nacional de Literatura de 1925 "al alimón" a Gerardo Diego (Versos humanos) y Rafael Alberti (Marinero en tierra), decisión que si por un lado aplaude, en lo que tiene de aliento para la nueva generación, por otro lado lamenta, "the scarcely veiled effort of the latter [young poets], in order to make themselves acceptable to the jury, to approach it in their turn, in an itch to adapt themselves to its benevolent, well-disposed, but, in general, a trifle out-of date, criteria, does not seem to me a good thing" (p. 358).

Otra de las críticas que de entrada dirige a la joven generación es su falta de cosmopolitismo, o, por lo menos, la presencia de un arraigado nacionalismo que se compadece mal con los ideales europeístas de la tribuna que acoge su comentario. $\mathrm{Si}$ bien es cierto que los jóvenes escritores siguen lo que ocurre en la cultura literaria de otras naciones, no deja de tener prioridad la relación con el paisaje, con el entorno cercano: "The landscape is imposed on the Spanish poet -in a poetical projection, of course- and he always appears to be incorporated in it" (p. 359). Los maestros de la joven generación lo son no sólo por su obra sino por la geografía que les acompaña: Juan Ramón Jiménez y Andalucía, Antonio Machado y Castilla, Moreno Villa y Ramón desde Madrid, o Alonso Quesada desde Canarias. El paisaje no deja de ser una formulación más de la "circunstancia" orteguiana.

Marichalar se atreve a establecer una jerarquía de la joven generación ordenada según la mayor o menor profundidad de los autores, y entiéndase aquí por "profundidad" y "ligereza" la mayor o menor afinidad con el clasicismo que se propone desde The Criterion y su hermana la Revista de Occidente. El canon "clasicista" es el siguiente: Salinas, Guillén, Alonso, Chabás, Diego, Lorca y Alberti.

Pero el comentario de Marichalar se centra sobre todo en los ya mencionados premios nacionales. De Diego prefiere su "old modern manner” (p. 360), aquélla cercana a la deshumanización 
de Ortega y advierte que el clasicismo de Diego en Soria o Versos humanos puede conducir al mismo colapso sufrido en Francia con Plain chat de Cocteau: resentirse de los peligros de la tendencia moderna más retórica y caer en el ornamentalismo. La conclusión del de Montesa es que cuando Diego escribía versos creacionistas tenía una relación más fluida con los clásicos.

Al llegar a Alberti, "the youngest and most notorious of our poets" habla con cierta sorna de su éxito: "his first book was an immediate and triumphant success, like the call of a trumpet in space" (p. 361). Según el canon antes transcrito, Alberti es el más ligero de entre los jóvenes poetas. De ahí no se moverá Marichalar ya que no existe otro argumento en su comentario sobre Alberti que no sea echar en falta cierta gravedad, o ridiculizar la figura del poeta alegre como un pájaro: "this open eagerness to please that offers an eternally smiling appearance in a laughing landscape vexes and irritates me in the end... For the modern man, a bird singing in the boughs is somewhat stupid as a singer" (loc. cit.).

A partir de la siguiente crónica, junio de 1927 (V, núm. 1), el Marqués de Montesa es un hombre enamorado del pasado ${ }^{16}$, lo que no quiere decir que deje de interesarle la actualidad de las letras españolas de su tiempo. De hecho le interesa y mucho, pero desde el punto y hora en que las novedades actualizan tradiciones. Esta mirada hacia atrás comienza, naturalmente, con la inevitable crónica sobre Góngora en su gran año. Marichalar arrima al sevillano hacia las discusiones sobre la poesía pura y le encuentra parientes europeos en Donne y Marino, aparte de la pervivencia de la tradición culta que evidencian los Sonetos espirituales de Juan Ramón. Nada nuevo. Lo que sí sorprende es que si a Alberti le pedía profundidad en la crónica anterior, ahora Góngora, y concretamente dos versos: "Los delfines van nadando / por lo más alto del agua", le sirve de pretexto para elaborar toda una teoría sobre la necesaria superficialidad del quehacer poético. "Poetry is felt, and to feel you need not to go too deeply" (p. 95), afirma. La crónica se cierra informando sobre lo que la nueva generación de poetas y profesores españoles prepara en torno al autor de las Soledades, resaltando, sobre todo, el trabajo filológico en ciernes: "Good critical editions and anthologies are lacking” (p. 98).

${ }^{16}$ La expresión la tomamos de un ensayo de W. Lewis sobre Pound titulado "A man in love with the past". 
Las últimas páginas que Marichalar remite a la revista de Eliot durante su primera etapa están dedicadas a la figura mítica y médica de Don Juan (VI, núm. 4). Médica por lo que respecta a los diagnósticos de Marañón y de Lafora, mítica por la rentabilidad de su arquetipo en la literatura y el pensamiento español de aquellos años. El cronista repasa la vigencia del mito de Don Juan en las letras españolas desde Valle a los Machado, pasando por el poema de Bergamín, "one of the most gifted minds of the modern generation" (p. 354), "Don Juan", incluido en el libro Enemigo que huye. También aquí tercia Ortega al utilizar la figura de Don Juan como símbolo de la filosofía vitalista, dechado de la espontaneidad que se opone a la razón socrática: "so the myth is saved!" (p. 355), exclama Marichalar, al reseñar la "salvación" de Ortega.

Desempeñan el papel protagónico de la crónica sobre Don Juan los análisis médicos de la patología donjuanesca de Lafora y Marañón, que sirven de pretexto a Marichalar para denostar y alabar determinadas posturas críticas. Lafora es el crítico objetivo, riguroso pero falto de fervor y por tanto de ironía (filisteo es el adjetivo que se gana) y Marañón es un crítico intuitivo y apasionado; mientras Lafora pretende desmitificar, Marañón pretende comprender. La identidad afeminada que oculta Don Juan y su falta de inteligencia, tesis centrales del estudio del Marañón, son los argumentos que Marichalar repite sin ponerlos en cuestión. De Lafora le molesta su irrespetuosidad religiosa, su desmitificación de Don Juan no a partir de rasgos psicológicos intrínsecos, sino recurriendo a su españolidad y trasfondo religioso. El libro de Lafora en el que se incluye el ensayo sobre Don Juan trataba también el tema del cubismo, hecho al que se ase Marichalar para recordarle una frase de Reverdy "a cubist poet - the best of them- and what is worse, an orthodox Catholic" (p. 350): “...de ce qui nous vient de Dieu, ce qui est le moins croyable c'est le naturel".

\section{$1928-1939$}

$\mathrm{Al}$ adentrarnos en la segunda mitad de 1928, The Criterion afronta su etapa menos literaria, que va a extenderse hasta los umbrales de la Segunda Guerra Mundial. Hacia la mitad de esos años difíciles, Antonio Marichalar reflexionaba de este modo sobre el cariz de los tiempos: "Se habla mucho de crisis —evidente-; 
se habla mucho de muerte - problemática-: del fin de una cultura. Y, sin embargo, empiezo a sospechar que allá, en la lejanía, todo eso va quedando definitivamente colocado, va encontrando su lugar y su fórmula. Por ello data. Y que yo soy, en cambio, el único que pasa"17.

Con tal incertidumbre de fondo, la presencia española en la revista de Eliot se afianza, sobre todo a través de las perspicaces crónicas del biógrafo de Osuna. Ello constituye la mejor muestra de la madurez paulatina de nuestra cultura literaria en la prueba de fuego que aporta las relaciones internacionales en una época de internacionalización como la que nos ocupa y precisamente antes de que los conflictos armados del final de los años treinta acaben con la modernidad.

Ahora bien, este tramo del recorrido por las referencias hispanas en la tribuna por antonomasia del modernism ha de abrirse con una reticencia, quizá no del todo retórica. El mismo año en que aparece su obra Manuel de Falla (1929) J. B. Trend publica un apasionado elogio de los ballets El sombrero de tres picos y, principalmente, El amor brujo. Ambos se encuadran en lo que el crítico llama el estilo "post-Diaghilev", como también por ejemplo algunas producciones coetáneas alemanas (VIII, núm. 32, p. 481), y de forma más precisa dentro de la producción del autor gaditano en su manera española. En El amor brujo la alianza de Falla y de Antonia Mercé, La Argentina, para Trend la mejor bailarina española viva (p. 483), logra "one of the greatest masterpieces of modern times" (p. 484). El entusiasmo del calificado espectador desemboca en el siguiente juicio: "Where is the boasted mundo latino, the «defence of the west», or, for that matter, Europe itself!... Away with all this Latin sophistication! Here we get down to the beggarly elements; and the only «Latin» quality of Falla's music is that it has enabled us to see these things so clearly" (p. 485).

El quiebro final hacia la claridad, definitoria de Juan Ramón Jiménez, de Ortega o de Guillén, evita un enfrentamiento en

17 Mentira desnuda. (Hitos), p. 7. Vale la pena traer a colación unas palabras de Edmund Wilson y sus amigos sólo un año anteriores a las que escribe Marichalar en 1933: "La crisis del mundo presente... es algo más que una mera crisis política y económica; y no se superará cuando se supere la depresión. Es una crisis de la cultura humana. Hoy nos enfrentamos con la necesidad imperativa de nuevas formas sociales, nuevos valores, un nuevo orden humano" (F. Kermode, Historia y valor. Ensayos sobre literatura y sociedad, trad. N. Catelli Quiroga, Península, Barcelona, 1990, p. 60). 
última instancia entre lo español y lo europeo que la misma "Music chronicle" del hispanista melómano hubiera desmentido de entrada.

En el cuerpo de la revista, dejando para luego las importantes secciones de reseñas de publicaciones periódicas y libros, sobresale la atención prestada a ilustres figuras de nuestra historia literaria y artística. Eso es así en los informes del Marqués de Montesa, pero sucede con otras colaboraciones. $\mathrm{Al}$ respecto, el trabajo más extenso de tema español que sale a la luz en The Criterion corre a cargo de Robert Sencourt y versa sobre San Juan de la Cruz. Nos hallamos ante un asunto que interesó profundamente al director de la publicación, que no en vano se había declarado en 1928 "anglocatólico" en religión, es decir miembro de la Iglesia de Inglaterra, y que editará en su revista varias noticias acerca de textos vinculados con la mística ${ }^{18}$.

El ensayo de Sencourt cuenta con una bibliografía crítica, obras de Jean Baruzi, Bruno de Jésus-Marie y Crisógono de Jesús Sacramentado, que demuestra su buen conocimiento de los estudios contemporáneos sobre el creador del Cántico y en la que destaca el título del P. Crisógono de Jesús San Juan de la Cruz: su obra científica y su obra literaria (El Mensajero de Santa Teresa, Madrid, 1929, 2 ts.), "one of the most valuable works on mysticism that has appeared in our time: it is the one book of outstanding value on St. John of the Cross" (X, núm. 41, p. 645). Con estas herramientas, Sencourt recorre desde la biografía de San Juan hasta su estilo, pasando por observaciones sobre la naturaleza del amor o la proximidad de las tareas del artista y el místico: "Each has the gift of being absorbed in admiring contemplation: each thirsts and longs to know that which he admires: each does this with such peculiar effort that spells of acute exhaustion, sometimes prolonged, seem to dull and deaden his faculties" (p. 647).

Sea como fuere, sólo le atañe al presente estudio la vertiente literaria del personaje y, en torno a ella, el punto de llegada de Sencourt es la metáfora, pues sobre los magistrales poemas del de Fontiveros comenta: "There is hardly a line that is not a metaphor" (p.651). No podemos olvidar que la metáfora supone

18 Sobre la relación directa entre Eliot y San Juan de la Cruz, relación de carácter textual, véase A. Soria Olmedo, "San Juan de la Cruz y la literatura contemporánea (1856-1942)", San Juan de la Cruz y la literatura de su tiempo, Junta de Andalucía-Turner, Madrid, 1991, pp. 50 ss. 
un instrumento privilegiado para los modernos ${ }^{19}$ y que las incursiones en el pasado que hemos visto y veremos están determinadas por el presente de partida y adquieren sentido en función de ese presente.

Además de las crónicas de Marichalar, el único texto español traducido en esta etapa de The Criterion pertenece a la Soledad I de don Luis de Góngora, obviamente ya bien pasadas las algazaras del centenario y cuando la vigencia de la estética gongorina había cedido considerable terreno en nuestras letras. Según nos informa Edward Meryon Wilson al reeditar su traducción del conjunto de dicha obra ${ }^{20}$, preparó la versión inglesa de las Soledades durante los años 1928 a 1930, y tras los intentos vanos de Eliot por encontrarle un editor, apareció la primera salida del libro en The Minority Press de Cambridge. El fragmento que adelantó The Criterion en julio de 1930 corresponde a los versos 182 a 228 de la edición que Dámaso Alonso publicó en 1927 bajo el sello de Revista de Occidente. En la nota que acompaña a esta traducción parcial, Wilson aclara: "The text I have followed is thus that of Gongora's early version, here at least, superior to the alterations made by him to comply with the censures of his friend Pedro de Valencia" (IX, núm. 37, p. 605), antes de hacer constar que su traducción no aspira a ser literal. En cuanto al pasaje del poeta barroco elegido, tiene una evidente unidad de significado y hay que reconocer el acierto del título sugerido por el hispanista británico: "Landscape and a moral". En efecto, a una visión de la naturaleza en todo su esplendor puesta al servicio del hombre siguen unas meditaciones sobre las glorias pasadas y el consuelo concedido por el tiempo. Si no la dificultad, al menos determinadas implicaciones éticas de los versos gongorinos todavía tenían algo que enseñar en la tierra del extraordinario predicador y poeta John Donne.

Pero hemos de abordar ya la tarea del cronista oficial de la cultura española en The Criterion a lo largo de los años treinta,

${ }^{19}$ R. Gómez de la Serna certifica: "La metáfora es, después de todo, la expresión de la relatividad. El hombre moderno es más oscilante que el de ningún otro siglo, y por eso más metafórico. Debe poner una cosa bajo la luz de otra. Lo ve todo reunido, yuxtapuesto, asociado... Lo único que quedará, que en realidad ha quedado, de unos tiempos y de otros ha sido la gracia de las metáforas salvadas" (Greguerías. Selección 1910-1960, ed. C. Nicolás, Espasa Calpe, Madrid, 1991, p. 51).

20 The Solitudes, trans. E. Meryon Wilson, Cambridge University Press, Cambridge, 1965, p. ix. 
esto es, el mismo historiador y homme de lettres de hasta entonces, el Marqués de Montesa, Antonio Marichalar. Su primer apunte en esta etapa contiene una pregunta que evidencia la capacidad de autocrítica del personaje, tan "modernista" en cada una de sus manifestaciones "I am wondering whether so marked and exceptional an interest in figures of the past, in contrast to indifference and neglect which are the portion of present-day production, is not something to be disturbed about" (VIII, núm. 30, p. 122). Ha aludido a las celebraciones de los centenarios de Góngora y de Goya en España. Ahora bien, de las siete crónicas que a partir de ese momento envía a la revista, Marichalar dedica tres a creadores de otro tiempo. Sólo que nuestro corresponsal, como dije arriba, no se regodea en ningún tipo de presunta "edad de oro", sino que sitúa a sus artistas pretéritos en la tradición moderna. De modo que Francisco de Goya, el protagonista del citado escrito, va a relacionarse con Baudelaire, con los impresionistas, con Picasso y, claro, con Ramón Gómez de la Serna, que acababa de dedicar al pintor el volumen Goya (1928): "This modern writer, who has some of the first fruits of the new literature to his credit, had once to deny the imputation of certain influences, and declared that he had been influenced by no one except Goya" (p. 126).

Pasan casi tres años hasta la siguiente "chronicle" (X, núm. 40). Al frente de ella incluye una introducción relativamente larga sobre las dificultades que conlleva la labor de analizar la literatura actual: "Just realize the utter thanklessness of his mission. He has to single out and appraise a movement without for one moment holding it up in its course. He has to comment upon something that is rapidly becoming past history" (p. 500). Tan agudo comienzo vale como captatio benevolentiae, mas también como autoparodia y como mecanismo que relativiza sus juicios. Lo más curioso es que quizá, a la postre, Marichalar, "a professional false-witness fully convinced of his own guilt" (loc. cit.), habla demasiado en serio sobre un trabajo de validez dudosa y por lo demás ingrato. De cualquier forma no vuelve a mandar a Londres una "crónica española" con tan peligrosas características como la que así entraba en fuego.

El diagnóstico ulterior sortea con eficacia ese cúmulo de asechanzas y la mejor prueba de ello consiste en lo bien que han resistido el paso del tiempo los nombres congregados por el de Montesa, sobre todo si tenemos en consideración que en determinados momentos ha de arriesgarse claramente. Algunas ex- 
cepciones confirman dicho planteamiento general, como por ejemplo la excesiva atención otorgada a Azorín, a la vez que no parece hacer bastante justicia a Valle a pesar de la opinión favorable de su crónica inaugural en The Criterion (p. 501). Por otra parte, los intentos de clasificación que realiza el crítico pecan de cierta confusión al acercarse a la literatura más reciente, aunque es obvio que a este respecto sus palabras preliminares cobran especial valor. Estamos ante matices susceptibles de discutirse que en absoluto menoscaban el alcance de un magnífico ejercicio interpretativo que, aplicado a su "generación", conduce a esta especie de declaración de principios: "it is made up of minds whose judgment is sound, whose approach to a subject is masterly, whose artistic conscience is both eclectic and constructive" (p. 503). Los adjetivos "ecléctica" y "constructiva" no sólo unen las obras de compañeros de fatigas literarias como Pedro Salinas, Jorge Guillén, José Bergamín, Dámaso Alonso, Vicente Aleixandre o Federico García Lorca a la del propio Marichalar, sino que definen con precisión la esencia de la modernidad en España.

El final del trayecto en 1931 por fuerza ha de ser político: "the idea of literature serving the purpose of revolution has also taken root at home, stirring up a group of turbulent journalists" (pp. 503-504). La distancia que toma el autor en relación con el fenómeno más característico de la vida cultural de los treinta apunta en dicha alusión y se confirma en su próxima "Spanish chronicle": "It is hardly possible to speak of literary life in Spain during the last few months, without showing how closely it is related to the world of politics... The real intellectual does not cease to be one. His vocation is that of an artist, although his diversion may be politics" (XI, núm. 43, p. 296) ${ }^{21}$.

Esto se concreta en Unamuno, Ortega o Marañón, casos distintos a Azaña, que en rigor le parece un político disfrazado de literato (p. 297). En consecuencia, Marichalar se desentiende

${ }^{21}$ Nos hallamos ante un leitmotiv del ensayo coetáneo, como demuestran A. P. Debicki, Spanish poetry of the twentieth century. Modernity and beyond, The University Press of Kentucky, Lexington, 1984, p. 53, o, con un enfoque más amplio, M. Hamburger, La verdad de la poesía. Tensiones en la poesía moderna de Baudelaire a los años sesenta, trads. M. A. Flores y M. Córdoba Magro, F.C.E., México, 1991, p. 181, sobre el que MARICHALAR vuelve en Mentira desnuda, por ejemplo, en p. 26: "Riñen artistas y políticos (como ahora comunistas y poetas del suprarrealismo), impidiéndoles una mutua inteligencia su distinta visión respectiva. El poeta no acepta ese punto de mira utilitario del político, y éste rechaza el idealismo del poeta porque es adverso a su concepto materialista del mundo". 
de esa parte de la producción literaria coetánea que no le interesa. Así describe las señas de identidad institucionistas de los intelectuales españoles (p. 299), alaba rozando la hipérbole a Ortega, "the man whose pronouncements excite the greatest expectation, both in Spain and Spanish America" (p. 301) y hasta se atreve a proponer una explicación para la llegada de la República. En este asunto es preciso reconocer que Marichalar, tan lúcido en sus apreciaciones estrictamente librescas, no acierta tanto a la hora de explicar la realidad socio política nacional, pues pretende dar cuenta de la caída de la monarquía a partir del individualismo de sus compatriotas, superado en una acción al modo de Fuenteovejuna (p. 302).

Las crónicas no vuelven a enfrentarse con la producción literaria española de esos años. Su creador expuso una coartada habilidosa: "How can I discuss, in these chronicles, a literature which remains inaccessible to most English readers? I see myself reduced to merely giving information, and I am opposed to that kind of writing" (XII, núm. 47, p. 250). No vale la pena replicar que antes fue capaz de escribir colaboraciones no exclusivamente informativas sin contar con que los textos comentados estuviesen traducidos, ni que es curioso que su decisión coincida con un momento de profunda discrepancia con el rumbo comprometido de las bellas letras, no sólo vigente en España. En cualquier caso, a continuación centra sus esfuerzos en valorar dos títulos últimamente trasladados al inglés: Our father San Daniely The revolt of the masses. La novela de Gabriel Miró es considerada dentro de la categoría que Marichalar llama "poesía en prosa" (Mentira desnuda, p. 19) en la línea de un Marcel Proust o de una Virginia Woolf, y antes de concluir dice: "Miró left some splendid books, if he did not succeed in producing good novels" (p. 254). En cuanto al volumen de Ortega, apenas si atiende a la pésima labor del anónimo traductor. Marichalar parte de la premisa "The style is the man, and in this case the persuasive expression of this thought in all its strictness" (loc. cit.), pero en cualquier circunstancia hubiese sido censurable suprimir metáforas y hasta pasajes enteros.

Las dos entregas inmediatamente posteriores del aristócrata a The Criterion exploran la senda de los clásicos. Marichalar aborda el tercer centenario de la muerte de Lope con el volumen Lope de Vega y su tiempo de Carl Vossler como base ${ }^{22}$. Una

${ }^{22}$ La versión española de la obra, que en alemán se había publicado en 1932, apareció un año después editada por Revista de Occidente. 
vez más el ensayista se nos muestra plenamente consciente de sus actos: "it is a well-known fact that the welcome extended to any literary or historical 'revenant' distinctly varies in proportion to the particular trend of contemporary historical events, as also to the particular critical norm that happens to be in fashion at the moment" (XIV, núm. 56, p. 457).

Es decir, a Marichalar le importa sobre todo un Lope vinculado a "nuestro tiempo". Dos hilos de unión viene a subrayar, uno estrictamente literario y más bien moral el otro: "We must also not forget to show how extraordinarily 'modern' Lope can be at moments, and also to what a degree certain passages of his writings suggest those from the works of some of our contemporaries" (p. 466), entre otros Proust, Gide o Joyce. Como vimos arriba, el de Montesa no va a la historia por ella misma, sino para iluminar el presente, un presente que conforme nos adentramos en la década de los años treinta se torna más y más incierto. La conclusión del artículo lopesco resulta ejemplar en este sentido, y con ella surge el segundo hilo mencionado. El Fénix reúne los contrarios, e incluso los supera a lo largo de su trayectoria vital y literaria (pp. 463 ss.); pues bien, en sus huellas recala Marichalar desde una época de escisión del país que le lleva a desear: "God grant that he [el español de hoy] may find the reflection of his country a thing of beauty, an image of calm self-possession at peace with herself and the world - as he would like to see his own reflected self, in other words" (p. 470).

Cuando ya la guerra civil destroza al país, en octubre de 1936, nuestro crítico publica en The Criterion un trabajo sobre Garcilaso y Bécquer. Para empezar, continúa perfilando su teoría personal sobre los imprecisos límites de antiguos y modernos:

For the classic writers were, in their day, bold and in advance of their age and they felt the urge to "write modern", and this is perhaps why Góngora and Lope de Vega have found, during their recent and respective centenaries, the greatest measure of love and esteem not only from the poet and the professor, but from among the most advanced and unruly in the younger generation (XVI, núm. 62, p. 90).

La modernidad que encarnan Ortega, Marichalar, Salinas o Diego, pero también, claro, Eliot o Pound, a la postre pasa por los nombrados escritores barrocos. Ahora bien, en 1936 el azar de los centenarios reúne a otros dos autores igualmente capaces de sustentar una lectura actual, Garcilaso y Bécquer. Sobre 
el aventajado discípulo de Petrarca, Montesa sentencia: "modern Spanish poetry takes its origin from the work of this «Europeanizer»" (p. 94); ahora bien, con algunos ajustes su dictamen vale igualmente para el seguidor de Heine. La coincidencia puede deberse a que ambos comparten la concepción del hecho poético como "contradictory synthesis" (p. 100) o como "mentira desnuda" 23 , según reza el título de la capital colección de ensayos que Marichalar publica en 1933. Por otra parte, convendría tenerse en cuenta la posibilidad de que tras semejante teoría, el escritor además esté proponiendo, a modo de respuesta a los males de su patria, un atisbo de utopía conciliadora: "Every day, I believe more firmly, that the world is working for a Christian identification of principles that are diametrically opposed to each other, although it may employ in the act the cruel process of dialectics" (p. 99). El único problema radicaba en que a esas alturas la "crueldad" dominaba por completo sobre la "identificación".

La última aparición de Antonio Marichalar en la revista significa un homenaje incondicional a José Ortega y Gasset en el análisis de dos textos recientes: "History as a system" (1935) y el "Préface pour le lecteur français" (1937) incluido en la traducción francesa de La rebelión de las masas. Estas obras "are the most closely-knit and the most forceful that this Spanish philosopher has written for a long time -I was going to say, that he has written during the whole of his literary career up to the present moment" (XVII, núm. 69, p. 707). Una explicación plausible para tal opinión, al menos una pizca exagerada, pasaría por hacer constar que Ortega en dichos trabajos aspira a situarse al nivel de las difíciles circunstancias del entorno europeo ${ }^{24}$, "one of the saddest moments in History” (p. 716) dirá Marichalar y

23 " «Cuando el hombre ha querido sustituir la marcha, ha creado la rueda, que no se parece en nada a la pierna». Para mí, esta frase de Apollinaire explica cabalmente la mentira desnuda del arte moderno. Esa es la realidad última... esa realidad captada por el poeta, a través de la falsa apariencia, es la realidad auténtica" (Mentira desnuda, p. 29).

24 Ortega en Historia como sistema señala: "El hombre se pregunta: ¿qué es esta única cosa que me queda, mi vivir, mi desilusionado vivir? ¿Cómo ha llegado a no ser sino esto? Y la respuesta es el descubrimiento de la trayectoria humana, de la serie dialéctica de sus experiencias, que, repito, pudo ser otra, pero ha sido la que ha sido y que es preciso conocer porque ella es... la realidad trascendente. El hombre enajenado de sí mismo se encuentra consigo mismo como realidad, como historia" (Obras completas, Alianza Editorial-Revista de Occidente, Madrid, t. 4, p. 49). 
eso que lo peor estaba todavía por venir. Los mecanismos de defensa que sugiere el fundador de Revista de Occidente son la Historia y Europa, y tanto The Criterion como el Marqués de Montesa suscribían la propuesta.

$\mathrm{Al}$ ocuparnos de las noticias sobre otras publicaciones periódicas incluidas en la de Eliot a lo largo de su época final, sorprende comprobar que en el rico panorama español del momento se repara casi exclusivamente en Revista de Occidente ${ }^{25}$. Un reproche continuo hace Charles K. Colhoun, que se encargó de la totalidad de estos comentarios, a la revista madrileña, a saber, el papel excesivo de colaboraciones alemanas y foráneas en general (por ejemplo en XI, núm. 42, p. 578). De manera que en una de sus notas con más carga interpretativa indica irónicamente: "Unfortunately, Señor Ortega y Gasset still has to look outside the frontiers of Spain for contributors that are expected to pull their weight by sheer main force of erudition - he must have literally thousands of applicants east of the Rhine- with the result that, native talent often gains the precarious footing of grass between heavy slabs of granolithic paving" (XIII, núm. 53, p. 712).

La suficiencia que estas palabras revelaban hubiera debido estar respaldada por un mejor conocimiento de la realidad literaria en España, sin embargo no sucede así, como descubre Colhoun de inmediato al establecer que los vanguardistas españoles "have always been such implacable foes of anything that might savour of «españolismo» or «peninsularity»" (loc. cit.). Este error le hace considerar extraña la fantasía "genuinely Spanish" de Ramón (p. 714), pero tenía aún más cerca la producción de Antonio Marichalar, tan cosmopolita como enraizada en lo hispánico. Y es que una de las virtudes innegables de los escritos de Colhoun radica en su positiva y sagaz valoración de la tarea del protagonista de estas páginas.

He has a supple intelligence and his Spanish individualism is always tempered with a restraint that is definitely trans-Pyrenean, and so it is not surprising that we should again find him playing a leading part in the interpretation of the work of prominent French contemporaries for the benefit of his countrymen (XV, núm. 61, p. 774).

${ }^{25}$ Como mínima excepción puede verse el puñado de líneas concedido a La Gaceta Literaria, entre las que leemos: "this periodical has resolutely set out to be a 'gazette', whose smallest faits-divers are, in themselves, of as great interest as its more weighty contributions" (X, 1931, núm. 41, p. 783). 
En todo caso, el esfuerzo de Marichalar no se limitaba a los autores franceses contemporáneos, sino que se extendía a nombres clave de la literatura en inglés, y desde luego, también cumplió con solvencia esa función de canal de comunicación desde España hacia Europa, como aquí se ha demostrado cabalmente.

Por fin hemos de interesarnos por las reseñas de tema español que se encuentran en la década última de The Criterion. No estará de más señalar al respecto que, como en las informaciones acerca de otras revistas, hallamos una perfecta coherencia entre el contenido de esta sección y los artículos que conforman la parte central de la publicación eliotiana. En efecto, Colhoun (XIII, núm. 50) atiende a la magnífica biografía que Montesa dedica a los Duques de Osuna, Pedro y Mariano Téllez-Girón, sobre todo a este último, que encarna "la leyenda del rumbo elevado a categoría filosófica" 26 . Mientras que F. McEachran entrega dos apuntes bien distintos sobre sendos títulos notables en la bibliografía de Ortega y Gasset. El correspondiente a la versión inglesa de El tema de nuestro tiempo culmina su dura crítica con esta penetrante advertencia: "What the advocacy of vitalism and spontaneity really means under modern conditions, is the victory of the economic collective whole or dictatorship of the Fascist kind, to neither of which will the human spirit, as we know it in the West, willingly surrender" (XI, núm. 44, p. 535).

Antonio Elorza llamaba la atención no hace mucho sobre la importancia que posee el referido libro de Ortega a fin de documentar "el repliegue ideológico" del autor a principios de los años veinte (p. 137) y añade en la línea del reseñista británico: "Por un momento, el liberalismo resulta eclipsado en nombre de una doctrina de exaltación individual, no muy alejada del naciente fascismo" (p. 140). Ahora bien, McEachran cambia de registro completamente apenas medio año después al rendir cuentas de su lectura de The revolt of the masses ${ }^{27}$. Tras

${ }^{26}$ Marichalar, Riesgo y ventura del Duque de Osuna. (Ensayo biográfico), Espasa Calpe, Madrid, 1930, p. 132. Como sugieren esas palabras, estamos ante un texto con una destacada vertiente paródica, así, el autor llega a decir de los tiempos del romanticismo: "Las cosas llegan a su propia parodia, henchida y ampulosa" (p. 149).

${ }^{27}$ En relación con esta obra ha de tenerse presente que "más que ninguna otra, haría que su nombre fuese conocido por lectores extranjeros"; según R. GraY, José Ortega y Gasset. El imperativo de la modernidad, Espasa Cal- 
definir al literato español como "a keen individualist of the liberal tradition" (XII, núm. 46, p. 145), elogia la superación del nacionalismo mediante la revitalización de Europa que reclama (p. 146) ${ }^{28}$.

De la mano de dichos problemas hemos de adentrarnos en el retrato de la guerra civil que presentó The Criterion, un retrato de modo harto significativo reducido casi únicamente al campo de las reseñas. Con el punto de vista oficial de la publicación parece discrepar E. W. F. Tomlin al describir Anarchy of herarchy de Madariaga, "one of the most original political treatises of the post-war period" (XVI, núm. 65, p. 698). Al hilo de sus comentarios del libro, Tomlin deja caer una llamada bastante explícita a intervenir en conflictos foráneos, y sus palabras en la Europa de 1937 han de aludir en primera instancia a la Guerra de España:

It is true that some people, including the present Foreign Secretary, have suggested that what we need to do, in order to establish a lasting international peace, is to give up, and urge others to give up, all 'interference' with foreign powers, to adopt a policy of 'live and let live'... but this is not really to give up anything except the problem, nor does it involve any sacrifice but that of individual responsibility (XVI, núm. 65, p. 697).

Sea como fuere, Eliot y The Criterion no están a favor de ningún tipo de intervención, por lo menos en lo que respecta al caso español. Al director de la revista le interesa el debate, que debió ocupar por entonces a buena parte de la sociedad y de la intelectualidad occidental, pero no tanto como para dedicarle entero uno de sus comentarios. Su tesis es: "so long as we are not compelled in our own interest to take sides, I do not see why we should do so on insufficient knowledge: and even any even-

pe, Madrid, 1994: "tipifica el punto de vista de ciertos críticos europeos de la cultura del período de entreguerras" (p. 215).

${ }^{28}$ Otras reseñas dignas de mención que recogen cuestiones ya tratadas por los artículos son la muy elogiosa de SENCOURT (XI, núm. 44, 1932) sobre El Islam cristianizado del sacerdote y arabista Miguel Asín, en la que se hace una fugaz alusión a The third spiritual alphabet de Osuna; la del filósofo neotomista M. C. D'ARCY acerca del estudio doctrinal St. John of the Cross de Bede Frost (XVII, núm. 68, 1938) o la de Orlo Williams en torno a The origins of modern Spain de Trend, personaje habitual en nuestro estudio del que se dirá "as a guide to Spain, Mr. Trend has no equal in England" (XIII, núm. 53, 1933-34, p. 665). 
tual partisanship should be held with reservations, humility and misgiving" (XVI, núm. 63, p. 290). Al cabo, la distancia que toma Eliot en relación con la guerra es tal que sencillamente llega a no querer ver la realidad. Sólo así el genial poeta y crítico puede hacer una observación como "Whichever side wins will not be the better for having had to fight for its victory" (loc. cit.).

Teniendo en cuenta semejante planteamiento se entienden la recomendación de Colhoun en 1937 a los fundadores de la Segunda República: "they can only wait and suffer" (XVI, núm. 64, p. 572) o las reticencias del poeta y novelista Edwin Muir sobre Spain de Auden, obra basada en la experiencia de su autor como conductor de ambulancias en el bando republicano (XVII, núm. 66, p. 150), poco antes de remitir a Lament for the death of a bullfighter and other poems de Lorca con un juicio poco esperable, por obtuso: "Their aim seems to be to achieve imagery and music and nothing else" (p. 154). Llegamos al jalón postrero de la presencia española en la revista insignia del modernism, justamente en el año anterior a su desaparición. Se trata de la reseña de J. Middleton Murry sobre Aux origines d'une tragédie (1937) ${ }^{29}$ de Alfredo Mendizábal Villalba, con prefacio del filósofo tomista "liberal" Jacques Maritain. Murry concede más atención al prefacio que a la obra propiamente dicha y se compromete sin fisuras contra cualquier totalitarismo (XVII, núm. 69, p. 721). Es lástima que no concretase cómo podía afectar dicha postura a la existencia inmediata de los lectores, pero las dudas se iban a disipar a la fuerza definitivamente en los meses siguientes, cuando ya el sueño de la modernidad había terminado.

En resumidas cuentas, si para terminar nos fijamos en el papel asignado a Antonio Marichalar en este proceso, nuestra percepción no está exenta de matizados claroscuros, como no podía ser menos en un "moderno" de pro y aristócrata. Por edad resulta más joven que el patrón de vanguardistas que fue Gómez de la Serna, pero en sus crónicas inglesas no concede a la vanguardia atención excesiva. En rigor, alguien que buscase en las crónicas de Marichalar una idea aproximada de lo que fue la vanguardia española se llevaría un buen chasco. Más bien la mirada del crítico, de manera coherente con la línea editorial del vehículo de sus escritos, se encamina paulatinamente

${ }^{29}$ La obra se publicó en Londres traducida al inglés un año más tarde con el título The martyrdom of Spain. 
hacia autores establecidos o hacia la misma historia. Por otra parte, de la "nueva literatura" tan sólo Ramón aparece publicado en The Criterion. Los otros autores españoles son Luis de Góngora y el mismo Montesa. Y es que ciertamente frente a la llamada "deshumanización", a la masificación y a la globalización que caracteriza a las sociedades modernas hubo respuestas no sólo variadas, sino hasta contradictorias.

\author{
Miguel Gallego Roca \\ Universidad de Almería \\ EnriQue Serrano Asenjo \\ Universidad de Zaragoza
}

\title{
APÉNDICE
}

A [Ldington], R., "Literature and the Honnête homme", I (1923), núm. 4, p. 422.

G[olhoun], G[harles] K., "Spanish periodicals. Revista de Occidente", X (1930), núm. 38, 202-206.

__ "Spanish periodicals... La Gaceta Literaria", X (1931), núm. 41, 783-784.

_ "Spanish, Spanish-American and Portuguese periodicals... La Revista de Occidente [sic] ... La Gaceta Literaria”, XI (1931-32), núm. $42,578-579$.

_ "Spanish, Spanish-American periodicals. Revista de Occidente", XII (1932-33), núm. 46, 172-173.

Colhoun, Charles K., "Spanish periodicals. Revista de Occidente... Also received: Mediodia", XII (1932-33), núm. 48, 720-722.

"Books of the Quarter... The perils and fortunes of the Duke of Osu$n a$. By Antonio Marichalar. Translated by Harriet de Onis", XIII (1933-34), núm. 50, 163-164.

_ "Spanish and Spanish-American periodicals. Revista de Occidente... Also recieved. Cruz y Raya", XIII (1933-34), núm. 53, 712-716.

_ "Spanish and Spanish-American periodicals. Revista de Occidente", XV (1935-36), núm. 61, 773-774.

C[Olhoun], C[HARLes] K., "Books of the Quarter... The Spanish tragedy. By E. Allison Peers", XVI (1937), núm. 64, p. 572.

D'ARCY, M. C., "Books of the Quarter... St. John of the Cross. By father Bede Frost”, XVII (1938), núm. 68, 538-539.

E[LIOT], T. S., "The function of a literary review", I (1923), núm. 4, p. 421.

“The idea of a literary review", IV (1926), núm. 1, 1-6. 
—_A commentary. The European idea", VI (1927), núm. 2, 97-99.

—_A commentary", XVI (1937), núm. 63, 289-293.

F[LINT], F. S., "Spanish periodicals. Revista de Occidente", II (1923), núm. 5, 109-110.

__ "Spanish periodicals. Revista de Occidente", IV (1926), núm. 4, $812-813$

Gómez de la Serna, RAmón, "From The New Museum”, I (1923), núm. 2, 196-201 [trad. de F. S. Flint].

Góngora y Argote, LuIs, "A fragment from the Solitudes. Landscape and a moral”, IX (1930), núm. 37, 604-605 [trad. de Edward Meryon Wilson].

Hawkes, C. P., "Cherrero. A note on the mascarades of the Pays Basque”, IV (1926), núm. 2, 297-305.

Marichalar, Antonio, "Contemporary Spanish literature”, I (1923), núm. 3, 277-292 [trad. de Mde. S. A. Middleton].

"Madrid chronicle", IV (1926), núm. 2, 357-362.

"Madrid chronicle", V (1927), núm. 1, 94-99.

"Madrid chronicle. Don Juan", VI (1927), núm. 4, 348-356.

"Madrid chronicle", VIII (1928), núm. 30, 122-127 [trad. de F. S. Flint].

"Spanish chronicle", X (1931), núm. 40, 499-504 [trad. de C. K. Colhoun].

—_ "Spanish chronicle”, XI (1931-32), núm. 43, 296-303 [trad. de J. B. Trend].

"Spanish chronicle", XII (1932-33), núm. 47, 250-256 [trad. de J. B. Trend].

"Spanish chronicle. The tercentenary of Lope de Vega", XIV (1934-35), núm. 56, 457-470 [trad. de Charles K. Colhoun].

"Spanish chronicle. Gracilaso and Bécquer", XVI (1936), núm. 62, 90-102 [trad. de Charles K. Colhoun].

"Spanish chronicle. Ideas and beliefs of José Ortega y Gasset", XVII (1938), núm. 69, 707-716 [trad. de Charles K. Colhoun].

McEachran, F., "Books of the Quarter... Gosé Ortega y Gasset (The modern theme) [sic]", XI (1932), núm. 44, 532-536.

"Books of the Quarter... The revolt of the masses. Ortega y Gasset", XII (1932-33), núm. 46, 144-146.

"Books of the Quarter... The Spanish cockpit. By Franz Borkenau", XVII (1938), núm. 67, 348-350.

McG., T., "Books of the Quarter... The mystical doctrine of St. John of the Cross. Being an abridgement of his works as translated into English by David Lewis and revised by Dom Benedict Zimmermann, O. D. C. With and introduction by R. H. J. Steuart, S. J.”, XIII (1933-34), núm. 53, 709-710.

Monro, Harold, "Books of the Quarter. Englishmen, Frenchmen, Spaniards. By Salvador de Madariaga”, VIII (1928), núm. 31, 313-315. 
Muir, EDWIn, "Books of the Quarter... Lament for the death of a bullfighter and other poems. By Federico García Lorca. The original Spanish with an English translation by A. L. Lloyd... Spain. By W. H. Auden”, XVII (1937), núm. 66, 148-154.

Murry, J. Middleton, "Books of the Quarter. Aux origins d'une tragédie. Par Alfred Mendizábal. Preface de Jacques Maritain”, XVII (1938), núm. 69, 718-721.

Sencourt, Robert, "Books of the Quarter... Goya. By Lord Derwent", X (1931), núm. 40, 574-578.

__ "St. John of the Cross", X (1931), núm. 41, 637-653. "Books of the Quarter... El Islam cristianizado. By Miguel Asín Palacios... The third spiritual alphabet. By F. de Osuna”, XI (1932), núm. 44, 525-529.

Tomlin, E. W. F., "Books of the Quarter... Anarchy or hierarchy. By Salvador de Madariaga", XVI (1937), núm. 65, 694-698.

Trend, J. B., "The moor in Spanish music”, II (1924), núm. 6, 204-219.

—_Music. The Spanish idiom”, III (1925), núm. 12. "Music. Spain and Hungary", IV (1926), núm. 1, 154-160. "Music. Musicians and philosophers", IV (1926), núm. 2, 342-349.

_ "Books of the Quarter. El pensamiento de Cervantes. By Américo Castro", V (1927), núm. 1, 146-148.

T[REND], J. B., "Spanish periodicals. Revista de Occidente, Carmen, Litoral, Archivo Español de Arte y Arqueología”, VII (1928), núm. 4, 459-463. "Music chronicle. Falla and the ballet", VIII (1929), núm. 32, 480486.

_ "Books of the Quarter... Unromantic Spain. By Mario Praz", IX (1930), núm. 36, 572-573.

Williams, Orlo, "Books of the Quarter... The origins of modern Spain. By J. B. Trend”, XIII (1933-34), núm. 53, 662-665.

W[ILSON], E[DWARD] M[ERYON], "Books of the Quarter... Shakespeare en la literatura española. By Alfonso Par”, XV (1935-36), núm. 59, 361-362. 